\title{
Multi Parameters Golden Ratio and Some Applications
}

\author{
Seyed Moghtada Hashemiparast ${ }^{1}$, Omid Hashemiparast ${ }^{2}$ \\ ${ }^{1}$ Department of Mathematics, Faculty of science, K. N. Toosi University of Technology, Tehran, Iran \\ ${ }^{2}$ Department of Engineering, Tehran University, Kish Pardis, Iran \\ E-mail: hashemiparast@kntu.ac.ir \\ Received January 9, 2011; revised April 30, 2011; accepted May 3, 2011
}

\begin{abstract}
The present paper is devoted to the generalized multi parameters golden ratio. Variety of features like two-dimensional continued fractions, and conjectures on geometrical properties concerning to this subject are also presented. Wider generalization of Binet, Pell and Gazale formulas and wider generalizations of symmetric hyperbolic Fibonacci and Lucas functions presented by Stakhov and Rozin are also achieved. Geometrical applications such as applications in angle trisection and easy drawing of every regular polygons are developed. As a special case, some famous identities like Cassini's, Askey's are derived and presented, and also a new class of multi parameters hyperbolic functions and their properties are introduced, finally a generalized Q-matrix called $G_{n}$-matrix of order $n$ being a generating matrix for the generalized Fibonacci numbers of order $\mathrm{n}$ and its inverse are created. The corresponding code matrix will prevent the attack to the data based on previous matrix.
\end{abstract}

Keywords: Generalized Golden Ratio, Trisection, Q-matrix, Fibonacci, Lucas, Gazale, Casseni

\section{Introduction}

During the last centuries a great deal of scientist's attempts has been made on generalizing the mathematical aspects and their applications. One of the famous aspects in mathematics is called golden ratio or golden proportion has been studied by many authors for generalization from different points of view. In recent years there were a huge interest of modern science in the application of the Golden section and Fibonacci numbers in different area of engineering and science. An extensive bibliography of activities can be found in a paper by Stakhov [1,2] who has investigated the generalized principle of Golden section and its vast area of applications. The main goal of his attention is to state the fundamental elements of this subject i.e. description of its basic concepts and theories and discussion on its applications in modern science. In this connection he explains the idea of the creation of a new mathematical direction called mathematics of Harmony that moved to intend for the mathematical simulation of those phenomena and processes of objective world for which Fibonacci numbers and Golden Section are their objective essence which can influence on the other areas of human culture. Stakhov considers the harmony mathematics from sacral geometry point of view and its applications in this field [3]. Some authors consider the extension of the Fibonacci numbers to create a generalized matrix with various properties suitable for the coding theory $[4,5]$. Even they established generalized relations among the code matrix elements for all values of Fibonacci p-numbers with a high ability. Order-m generalized of Fibonacci p-numbers for a matrix representation gives some identities [6] useful for further application in theoretical physics and Metaphysics [7-10]. Some authors determine certain matrices whose parameters generate the Lucas p-numbers and their sums creates the continues functions for Fibonacci and Lucas p-numbers which is considered in [11-14] and their generalized polynomials and properties are also introduced in [15], based on Golden section the attempt of build up the fundamental of a new kind of mathematical direction is addressed in [3] which is the requirements of modern natural science and art and engineering, these mathematical theories are the source of many new ideas in mathematics, philosophy, botanic and biology, electrical and computer science and engineering, communication system, mathematical education, theoretical physics and particle physics [16].

Other area of applications returns to the connection between the Fibonacci sequence and Kalman filter, by exploiting the duality principle control [17], finally numerical results of generalized random Fibonacci se- 
quences which are stochastic versions of classical Fibonacci sequences are also obtained [18]. Recently Eduardo Soroko developed very original approach to structural harmony of system [19], using generalized Golden sections. He claims the Generalized Golden sections are invariant which allow natural systems in process of their self-organization to find the harmonic structure, stationary regime of their existence, structural and functional stability.

Following the introduction, in Section 2 we establish multi parameters Golden Ratio and geometric applications. In Section 3 we generalized the Gazale formula. Sections 4 and 5 investigate the generalized hyperbolic functions and double parameters matrices and applications.

\section{Preliminaries}

In this paper we start a geometrical discussion which leads to a generalized form of golden ratio with multi parameters that for the case of single parameter it changes to the ordinary generalized form which has been considered by authors in the recent years.

Suppose a line $\mathrm{AB}$ is to divided in two parts $\mathrm{AC}$ and $\mathrm{CB}$ (Figure 1) such that

$$
a \frac{A B}{A C}=\left(\frac{\beta A C}{C B}\right)^{n}
$$

where $a$ and $\beta$ are real positive numbers. Let

$$
\begin{aligned}
& \frac{\beta A C}{C B}=x, \text { then } \\
& \quad a \frac{A B}{A C}=\left[a \frac{A C+C B}{A C}\right]=a\left[1+\frac{C B}{A C}\right]=a+a\left(\frac{\beta}{x}\right)
\end{aligned}
$$

Thus we obtain

$$
\left(a+\frac{a \beta}{x}\right)=x^{n}
$$

or

$$
x^{n+1}=a x+b
$$

where $a \beta=b$. In a special case for $n=1$ we intend to divide $A B$ such that

$$
a \frac{A B}{A C}=\frac{\beta A C}{C B}
$$

then we have

$$
x^{2}-a x-b=0
$$

or

$$
x_{1}=\frac{a+\sqrt{a^{2}+4 b}}{2}
$$

and

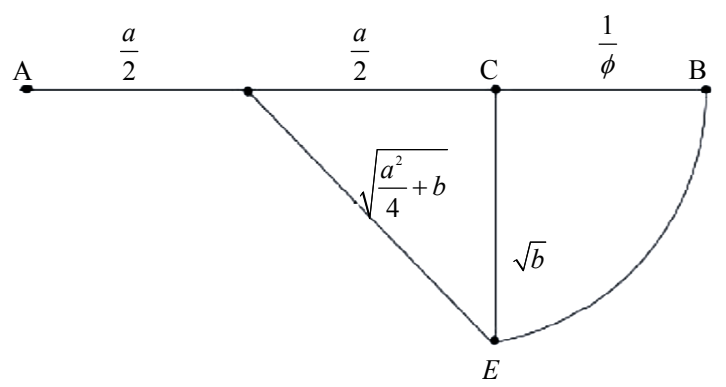

Figure 1. Dividing the line $A B$ in generalized Golden section.

$$
x_{2}=\frac{a-\sqrt{a^{2}+4 b}}{2}
$$

We call the positive root of this equation the generalized two parameters golden ratio $\phi(a, b)$

$$
\phi(a, b)=a\left[\frac{1}{2}+\frac{\sqrt{1+\frac{4 b}{a^{2}}}}{2}\right]
$$

for the case $b=1$ it gives a uniparameter $\phi_{a}$

$$
\phi_{a}=\phi(a, 1)=\frac{a}{2}+\frac{\sqrt{a^{2}+4 b}}{2}
$$

which is called generalized golden ratio and has been considered in recent years by some authors, for $a=1$ gives the famous historical golden ratio

$$
\phi=\phi_{1}=\phi(1,1)=\frac{1+\sqrt{5}}{2}
$$

where the ratio of the adjacent numbers in Fibonacci series and Lucas series tends towards this irrational number.

Let us consider the properties of this golden ratio, for $a=b$ we have

$$
\phi=a\left[\frac{1}{2}+\frac{\sqrt{1+\frac{4}{a}}}{2}\right]
$$

which is one solution of equation $x^{2}-a x-a=0$. Now other properties of the ratio can be considered. We have

$$
a+\frac{b}{\phi}=\phi
$$

the generalized golden ratio $\phi$ when $a=1$ reduces to the well-known historical golden ratio $\phi=\frac{1+\sqrt{5}}{2}$ which has many properties and application in art, engineering, physics and mathematics. Similar properties can be es- 
tablished in the generalized case. For instance the generalized $\phi$ can be expressed in terms of itself, like

$$
\phi=a+\frac{1}{\phi}
$$

that can be expanded into this fraction or nested roots equations that goes on for ever and called continued fraction or nested roots

$$
\begin{aligned}
& \phi=\sqrt{1+\sqrt{1+\sqrt{1+\cdots}}} \\
& \phi_{a}=\sqrt{1+a \sqrt{1+a \sqrt{1+a \cdots}}} \\
& \phi(a, b)=\sqrt{b+a \sqrt{b+a \sqrt{b+a \sqrt{\cdots}}}}
\end{aligned}
$$

Using the $\phi$ relation in term of itself we get the following continued fractions for $\phi, \phi_{a}, \phi(a, b)$ respectively

$$
\begin{gathered}
\phi=\frac{1}{1+\frac{1}{1+\frac{1}{1+\cdots}}} \\
\phi_{a}=a+\frac{1}{a+\frac{1}{a+\frac{1}{a+\cdots}}} \\
\phi(a, b)=a+\frac{b}{a+\frac{b}{a+\frac{b}{a+\cdots}}}
\end{gathered}
$$

Stakhov and Rozin [20,21] give some interesting results for $n=2$ and $a=b=1$, similar results are verified for different values of $a$ and $b$, two dimension periodic continued fractions are considered by some authors $[22,23]$ using above formulas may generalize the application in art and architecture. The above line AB can be divided in $n$ sections and by the successive ratios a system of equations will be created such that for a given value $n$, (1) be extended to multi parameters ratios to create a multi parameters Golden ratio. In this paper we concentrate on a generalized double parameters and applications of this ratio.

$$
\text { If } a=b \text { in (3), then } \phi(a, a)=a\left[\frac{1}{2}+\frac{\sqrt{1+\frac{4}{a}}}{2}\right] \text {, the }
$$

generalized single parameter ratio different from $\phi(a, 1)$ studied by Stakhov, actually (2) can be considered as a characteristic function of a difference equation of order $n$ with parameters $a$ and $b$

$$
P_{n+2}(a, b)=a P_{n+1}(a, b)+b P_{n}(a, b)
$$

which can be generalized to a multi parameters cases.

$$
\begin{aligned}
& P_{n+p}\left(a_{1}, a_{2}, \cdots, a_{p}\right)=\sum_{i=1}^{p} a_{i} P_{n+p-i}\left(a_{1}, a_{2}, \ldots, a_{p}\right), \\
& p=2,3, \cdots
\end{aligned}
$$

Theorem 2.1 Let $A D B$ be an isosceles triangle with the vertex angle $\alpha$, then the necessary and sufficient conditions for the angle $B A C$ equals to $\alpha$ (see Figure 2) $(0<\alpha<\pi / 2)$, is dividing the $B D$ by $C$ in generalized Golden ratio, such that $D C=a$ and $B C=1 / \phi$ and $B C+C D=a+\frac{1}{\phi}$.

Proof Without losing the generality put $b=1$, traingle $A D B$ is isosceles and $\hat{A}=\hat{B}=(\pi / 2)-\alpha$, the condition is sufficient, because if $D C=a$ and $C B=1$ then $D B=D C+C B$ or $D B=a+(1 / \phi)=a \phi$. For the triangles $A D C$ and $A D B$ we have respectively

$$
\begin{aligned}
& A C^{2}=D A^{2}+D C^{2}-2 D A \cdot D C \cos (\alpha) \\
& A B^{2}=D A^{2}+D B^{2}-2 D A \cdot D B \cos (\alpha)
\end{aligned}
$$

or,

$$
\begin{aligned}
& A C^{2}=a^{2} \phi^{2}+a^{2}-2 a \cdot a \phi \cos (\alpha) \\
& A B^{2}=a^{2} \phi^{2}+a^{2} \phi^{2}-2 a \phi \cdot a \phi \cos (\alpha)
\end{aligned}
$$

If $A B=A C$ then the $A B C$ will be an isosceles triangle and $\widehat{A B C}=\widehat{A C B}=\frac{\pi}{2}-\frac{\alpha}{2}$ and also $\cos (\alpha)=\frac{\phi+1}{2 \phi}$, so we will have $\widehat{B A C}=\pi-\left[\left(\frac{\pi}{2}-\frac{\alpha}{2}\right)+\left(\frac{\pi}{2}-\frac{\alpha}{2}\right)\right]=\alpha$. On the other hand the condition is necessary, because if $\widehat{B A C}=\alpha$ then $\widehat{A C B}=\pi-\left[\left(\frac{\pi}{2}-\frac{\alpha}{2}\right)+\alpha\right]=\frac{\pi}{2}-\frac{\alpha}{2}$, hence $A C=A B$ and triangle $A B C$ will be isosceles and we conclude easily $D C=a, C B=\frac{1}{\phi}$.

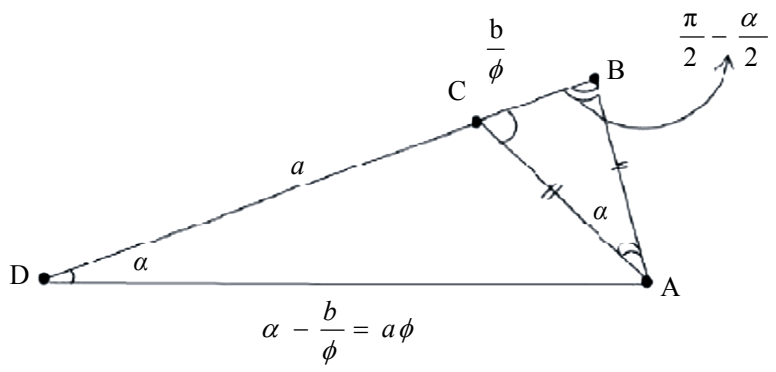

Figure 2. Dividing $B D$ by point $C$ in the generalized golden ratio. 
The impossibility proofs are so advanced, that many people flatly refuse to accept the problem are impossible [24]. The proofs of impossibilities of certain geometric constructions like doubling the cube and squaring the circle, trisecting the angle is impossible and details can be found in some references [25], trisecting the angle is impossible that is there exist an angle that cannot be trisected with a straightedge and compass. On the other hand there are some angles which may be trisected, some author give valuable discussion for ideal and classical trisection of an arbitrary angle [26]. Using generalized golden ratio one may establish the following criteria for trisection of a given angle.

Now, we construct an isosceles triangle $A D B$ whose vertex angle is $\widehat{A D B}=\alpha$ (Figure 3), hence the base angles are $\left(\frac{\pi}{2}-\frac{\alpha}{2}\right)$. By taking the line $B D$ and marking $C$ on the line in the generalized golden ratio, the angle $\widehat{B A C}=\alpha$, now we draw a circle with the center $D$ and radius $D A=D B=a+(1 / \phi)=a \phi$, then the line $A C$ will contacts with this circle at a point say $E$, the arc $E A B E=2 \alpha$. By a similar way we divide $D E$ in generalized golden ratio and find $C^{\prime}$ such that $D C^{\prime}=a$ and $C^{\prime} E=1 / \phi$, now the line $B C^{\prime}$ will contact with the circle in a point say $E^{\prime}$, joining $D E^{\prime}$ we have $\widehat{A D B}=$ $\widehat{B D E}=\widehat{E D E^{\prime}}$ The procedure can be carried on to produce a desired polygon by a proper selection of the angle $\alpha$. Thus we will have the following proposition for trisection of an arbitrary angle.

Proposition For trisection of a given angle first construct an isosceles triangle $A D B$ with vertex angle $3 \alpha$ and side $D B=D A=a \phi$ (Figure 4), then divide $D B$

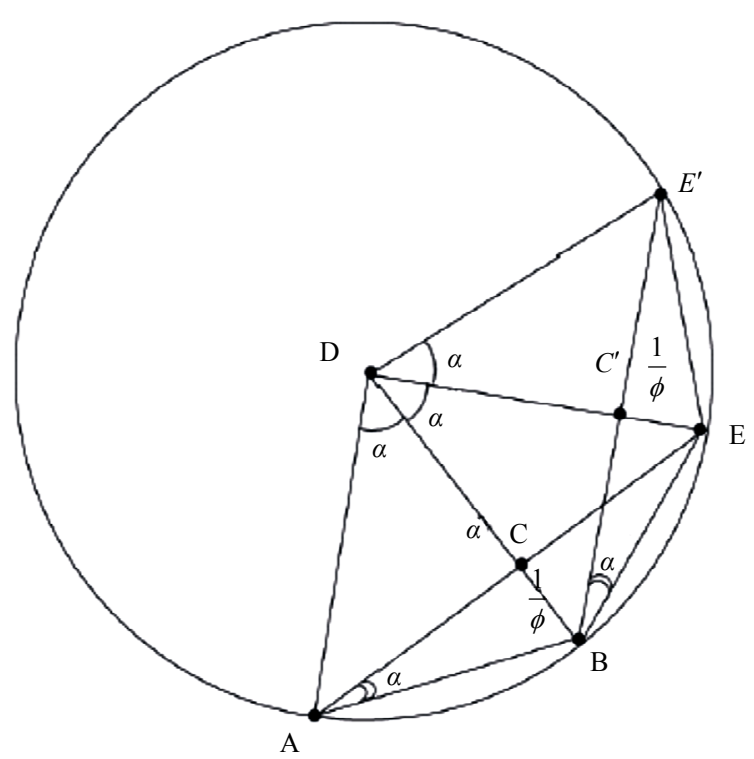

Figure 3. Trisecting the angle by using generalized golden section.

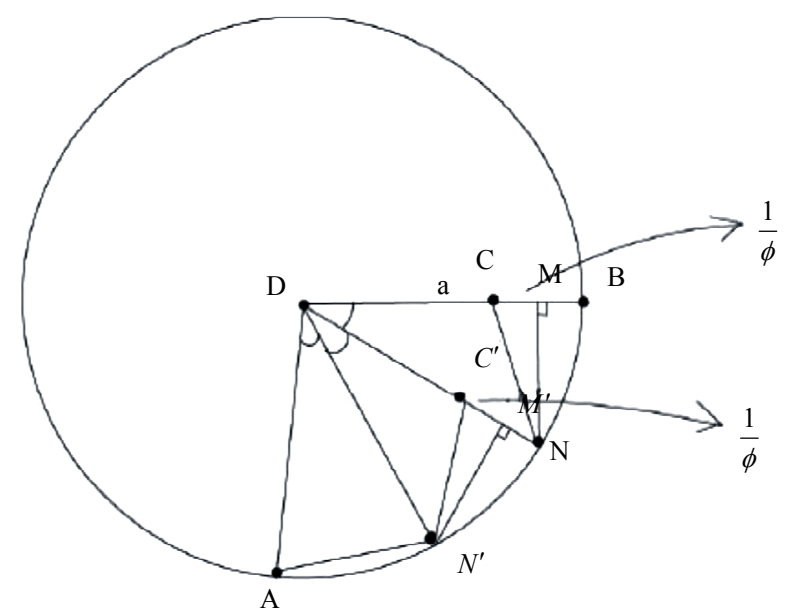

Figure 4. Algorithm for trisecting the angle by using generalized golden section.

into two parts $D C=a$ and $C B=1 / \phi$, and find $M$ the middle point of $C B$, draw a line per pendicula to $C B$ from $M$, which contact the circle at point say $N$, connect $D$ and $N$ by segment $D N$, then divide $D N$ into two parts $D C^{\prime}$ and $C^{\prime} N$ on golden ratio such that $D C^{\prime}=a$ and $C^{\prime} N=1 / \phi$ again find the point $M^{\prime}$ the middle point of $C^{\prime} N$ draw a line perpendicular to $C^{\prime} N$ at point $M^{\prime}$, extend $C^{\prime} N$ to contact the circle at point $N^{\prime}$, then draw $D N^{\prime}$, angle $3 \alpha$ will be trisected by $D N$ and $D N^{\prime}$.

When $D B$ is divided into two segment $a$ and $1 / \phi$, then $\cos (\alpha)=\frac{\phi+1}{2 \phi}$ we can easily calculate a with re-

spect to $\cos (\alpha)$, and we get

$$
a=\frac{1-b(2 \cos \alpha-1)^{2}}{(2 \cos \alpha-1)}
$$

having $\alpha, a$ can easily be calculated, for given $\alpha$ when the segments are $a$ and $b / \phi$ respectively, and are given in the Table 1 for $b=1$ and some nominal values of $\alpha$.

\section{Generalized Gazale Formula}

Let $a$ and $b$ be any real positive number, and define a Generalized Gazale sequence $P_{n}(a, b)=P, n=0,1,2, \cdots$ if and only if $P_{0}(a, b)=0$ and $P_{1}(a, b)=1$ and for all $n \geq 0$

$$
P_{n+2}(a, b)=a P_{n+1}(a, b)+b P_{n}(a, b)
$$

To attain the solution, using characteristics method gives the following quadratic equation

$$
\phi^{2}-a \phi-b=0
$$

where 
Table 1. Values of $\operatorname{Cos}(\alpha), a$ and $\phi$ for the nominal values of $\alpha$.

\begin{tabular}{llll}
\hline $\cos \alpha=\frac{1}{2}$ & $\alpha=60^{\circ}$ & $a=\infty$ & $\phi=\infty$ \\
\hline $\cos \alpha=\frac{\sqrt{2}}{2}$ & $\alpha=45^{\circ}$ & $a=2$ & $\phi=\sqrt{2}+1$ \\
$\cos \alpha=\frac{\sqrt{5}+1}{4}$ & $\alpha=36^{\circ}$ & $a=1$ & $\phi=\frac{\sqrt{5}+1}{2}$ \\
$\cos \alpha=\frac{\sqrt{3}}{2}$ & $\alpha=30^{\circ}$ & $a=\frac{3-\sqrt{3}}{2}$ & $\phi=\frac{\sqrt{3}+1}{2}$ \\
$\cos 20=0.940$ & $\alpha=20^{\circ}$ & $a=0.256$ & $\phi=1.136$ \\
$\cos 20=0.985$ & $\alpha=10^{\circ}$ & $a=0.061$ & $\phi=1.031$ \\
$\cos 0=1$ & $\alpha=0^{\circ}$ & $a=0$ & $\phi=1$ \\
\hline
\end{tabular}

$$
r_{1}=\frac{a+\sqrt{a^{2}+4 b}}{2}
$$

and

$$
r_{2}=\frac{a-\sqrt{a^{2}+4 b}}{2}
$$

are the roots of characteristics function and the positive root $r_{1}$ can be written as

$$
r_{1}=a\left[\frac{1}{2}+\frac{\sqrt{1+\frac{4 b}{a^{2}}}}{2}\right]=\phi(a, b)
$$

where $\phi(a, b)$ is the Generalized Golden Ratio and

$$
r_{2}=-a\left[-\frac{1}{2}+\frac{\sqrt{1+\frac{4 b}{a^{2}}}}{2}\right]=-\frac{b}{\phi(a, b)}
$$

Hence the general solution of (1) can be written as

$$
P_{n}(a, b)=C_{1}[\phi(a, b)]^{n}+C_{2}\left[\frac{-b}{\phi(a, b)}\right]^{n}
$$

Looking at the two initial values $P_{0}$ and $P_{1}$, the coefficients $C_{1}$ and $C_{2}$ can be determined, thus

$$
P_{n}(a, b)=\frac{1}{\sqrt{a^{2}+4 b}}\left\{\phi^{n}(a, b)-\left(-\frac{b}{\phi(a, b)}\right)^{n}\right\}
$$

For $b=1, P_{n}(a, 1)=P_{n}(a), \phi(a, 1)=\phi_{a}=\frac{1}{2}+\frac{\sqrt{a^{2}+4}}{2}$

$$
P_{n}(a)=\frac{1}{\sqrt{a^{2}+4}}\left\{\phi_{a}^{n}-\left(-\frac{1}{\phi_{a}}\right)^{n}\right\}
$$

which is the Gazale formula for the Generalized Fibonacci sequence, and if $F_{n}$ is the ordinary Fibonacci sequence, the general solution to

$$
F_{n+2}=F_{n+1}+F_{n}
$$

will be

$$
\begin{aligned}
F_{n} & =P_{n}(1)=\frac{1}{\sqrt{5}}\left\{\phi_{1}^{n}-\left(-\frac{1}{\phi_{1}}\right)^{n}\right\} \\
& =\frac{\left(\frac{\sqrt{5}+1}{2}\right)^{n}-\left(\frac{1-\sqrt{5}}{2}\right)^{n}}{\sqrt{5}}
\end{aligned}
$$

for the case $a=1$, and for the case $a=2, P_{n}(2,1)$ gives the John Pell (1610-1685) formula, and this formula generates an infinite number of generalized Fibonacci number of different orders for $a$ and $b$.

The following Theorem is a generalized aspect of theorems that have been established for Fibonacci and Lucas numbers.

Theorem 3.1 If $n \geq 1$ and $a, b$ are real positive numbers, then

$$
P_{n+1}(a, b) \cdot P_{n-1}(a, b)-P_{n}^{2}(a, b)=(-b)^{n-1}
$$

and is independent of $a$.

Proof We have

$$
\begin{aligned}
& P_{n+1}(a, b)=\frac{1}{\sqrt{a^{2}+4 b}}\left\{\phi^{n+1}(a, b)-\left(-\frac{b}{\phi(a, b)}\right)^{n+1}\right\} \\
& P_{n-1}(a, b)=\frac{1}{\sqrt{a^{2}+4 b}}\left\{\phi^{n-1}(a, b)-\left(-\frac{b}{\phi(a, b)}\right)^{n-1}\right\} \\
& P_{n}(a, b)=\frac{1}{\sqrt{a^{2}+4 b}}\left\{\phi^{n}(a, b)-\left(-\frac{b}{\phi(a, b)}\right)^{n}\right\}
\end{aligned}
$$

Then

$$
\begin{aligned}
P_{n+1}(a, b) \cdot P_{n-1}(a, b) & =\frac{1}{a^{2}+4 b}\left\{\phi^{2 n}(a, b)+\left(\frac{b}{\phi(a, b)}\right)^{2 n}\right. \\
& \left.-\left(b^{n-1} \phi^{2}(a, b)\right)-\left(\frac{b^{n+1}}{\phi^{2}(a, b)}\right)\right\}
\end{aligned}
$$

and

$$
\begin{aligned}
& P_{n}^{2}(a, b) \\
& =\frac{1}{a^{2}+4 b}\left\{\phi^{2 n}(a, b)+\left(\frac{b}{\phi(a, b)}\right)^{2 n}-2(-b)^{n}\right\}
\end{aligned}
$$

Thus 


$$
\begin{aligned}
& P_{n+1}(a, b) \cdot P_{n-1}(a, b)-P_{n}^{2}(a, b) \\
& =\frac{(-b)^{n-1}}{a^{2}+4 b}\left\{\phi^{2}(a, b)+\frac{b^{2}}{\phi^{2}(a, b)}+2(-b)\right\} \\
& =\frac{(-b)^{n-1}}{a^{2}+4 b}\left\{\left(\phi(a, b)+\frac{b}{\phi(a, b)}\right)^{2}\right\} \\
& =\frac{(-b)^{n-1}}{a^{2}+4 b}\left(a^{2}+4 b\right)=(-b)^{n-1}
\end{aligned}
$$

Similar identities like the Askey's and Catelan's and d'Ocagne's can be generalized. For instance in Osler and Hilburn [2] Askcy's hyperbolic sine identity for ordinary Fibonacci sequence is given as

$$
F_{n}=\frac{2}{i^{n} \sqrt{5}} \sinh (n \log i \phi)
$$

and generalized form in [27,28] for real positive $a>0$ is

$$
G_{n}=\frac{2}{i^{n} \sqrt{a^{2}+4}} \sinh \left(n \log i \phi_{a}\right)
$$

If we generalize (11) for $P_{n}(a, b)$ we obtain

$$
P_{n}(a, b)=\frac{2 b^{\frac{n}{2}}}{i^{n} \sqrt{a^{2}+4 b}} \sinh \left(n \log i b^{-\frac{1}{2}} \phi(a, b)\right)
$$

where $P_{n}(a, 1)=G_{n}$ and $P_{n}(1,1)=F_{n}$.

Because we have

$$
\begin{aligned}
P_{n}(a, b) & =\frac{2}{i^{n} b^{-\frac{n}{2}} \sqrt{a^{2}+4 b}} \sinh \left(n \log i b^{-\frac{1}{2}} \phi(a, b)\right) \\
& =\frac{2}{i^{n} b^{-\frac{n}{2}} \sqrt{a^{2}+4 b}} \frac{e^{\log \left(i b^{-\frac{1}{2}} \phi(a, b)\right)^{n}}-e^{\log \left(i b^{-\frac{1}{2}} \phi(a, b)\right)^{-n}}}{2} \\
& =\frac{1}{i^{n} b^{-\frac{n}{2}} \sqrt{a^{2}+4 b}} \\
& \times\left(i^{n} b^{-\frac{n}{2}} \phi^{n}(a, b)-i^{-n} b^{\frac{n}{2}} \phi^{-n}(a, b)\right) \\
& =\frac{\phi^{n}(a, b)-(-b)^{n}(\phi(a, b))^{-n}}{\sqrt{a^{2}+4 b}} \\
& =\frac{1}{\sqrt{a^{2}+4 b}}\left\{\phi^{n}(a, b)-\left(-\frac{b}{\phi(a, b)}\right)^{n}\right\}
\end{aligned}
$$

Thus

$$
P_{n}(a, b)=\frac{2 b^{\frac{n}{2}}}{i^{n} \sqrt{a^{2}+4 b}} \sinh \left(n \log i b^{-\frac{1}{2}} \phi(a, b)\right) .
$$

\section{Golden Hyperbolic Functions}

Similar to the Stakhov and Rozin hyperbolic functions [20], we can use the same approach and introduce the generalized hyperbolic functions of order $(a, b)$ in the following form

$$
\begin{aligned}
P_{n}(a, b) & =\frac{1}{\sqrt{a^{2}+4 b}}\left\{\phi^{n}(a, b)-\left(-\frac{b}{\phi(a, b)}\right)^{n}\right\}, \\
P_{-n}(a, b) & =\frac{1}{\sqrt{a^{2}+4 b}}\left\{\phi^{-n}(a, b)-\left(-\frac{b}{\phi(a, b)}\right)^{-n}\right\} \\
& =\frac{1}{\sqrt{a^{2}+4 b}}\left\{-\phi^{n}(a, b)\left(-\frac{1}{b}\right)^{n}+\left(\frac{1}{\phi^{n}(a, b)}\right)\right\} \\
& =\frac{-(-b)^{-n}}{\sqrt{a^{2}+4 b}}\left\{\phi^{n}(a, b)-\left(-\frac{b}{\phi(a, b)}\right)^{n}\right\} \\
& =-\left(-\frac{1}{b}\right)^{n} \frac{1}{\sqrt{a^{2}+4 b}}\left\{\phi^{n}(a, b)-\left(-\frac{b}{\phi(a, b)}\right)^{n}\right\}
\end{aligned}
$$

Then we will have

$$
P_{-n}(a, b)=-\left(-\frac{1}{b}\right)^{n} P_{n}(a, b)
$$

We define

$$
\left\{\begin{array}{l}
S P_{n}=S P_{n}(a, b)=(-b)^{-\frac{n}{2}} \phi^{n}(a, b)-(-b)^{\frac{n}{2}} \phi^{-n}(a, b) \\
C P_{n}=C P_{n}(a, b)=(-b)^{-\frac{n}{2}} \phi^{n}(a, b)+(-b)^{\frac{n}{2}} \phi^{-n}(a, b)
\end{array}\right.
$$

Then, $S P_{n}=-S P_{-n}$ and $C P_{n}=C P_{-n}$.

$$
P_{n}(a, b)=\left\{\begin{array}{cc}
(-b)^{\frac{n}{2}} S P_{n} & n=2 k+1 \\
(-b)^{\frac{n}{2}} C P_{n} & n=2 k
\end{array}\right.
$$

where

$$
\phi(a, b)=a\left[\frac{1}{2}+\frac{\sqrt{1+\frac{4 b}{a^{2}}}}{2}\right]
$$

Theorem 4.1 Following recursive relation can be established

$$
P_{n+2}(a, b)=a P_{n+1}(a, b)+b P_{n}(a, b)
$$

\section{Proof}

$$
P_{n}(a, b)=\frac{1}{\sqrt{a^{2}+4 b}}\left\{\phi^{n}(a, b)-\left(-\frac{b}{\phi(a, b)}\right)^{n}\right\}
$$




$$
\begin{aligned}
P_{n-1}(a, b)= & \frac{1}{\sqrt{a^{2}+4 b}}\left\{\phi^{n-1}(a, b)-\left(-\frac{b}{\phi(a, b)}\right)^{n-1}\right\} \\
P_{n-2}(a, b)= & \frac{1}{\sqrt{a^{2}+4 b}}\left\{\phi^{n-2}(a, b)-\left(-\frac{b}{\phi(a, b)}\right)^{n-2}\right\} \\
a P_{n-1}+b P_{n-2}= & \frac{1}{\sqrt{a^{2}+4 b}}\left\{\phi^{n-2}(a, b)[a \phi(a, b)+b]\right. \\
& \left.-\left(-\frac{b}{\phi(a, b)}\right)^{n-2}\left(-\frac{a b}{\phi(a, b)}+b\right)\right\} \\
= & \frac{1}{\sqrt{a^{2}+4 b}}\left\{\phi^{n}(a, b)-\left(-\frac{b}{\phi(a, b)}\right)^{n}\right\} \\
= & P_{n}(a, b)
\end{aligned}
$$

Similarly we define

$$
\begin{aligned}
\operatorname{COTP}_{x}(a, b) & =\frac{C P_{x}(a, b)}{S P_{x}(a, b)} \\
& =\frac{\phi^{x}(a, b)+(-b)^{x} \phi^{-x}(a, b)}{\phi^{x}(a, b)-(-b)^{x} \phi^{-x}(a, b)} \\
& =\frac{\phi^{2 x}(a, b)+(-b)^{x}}{\phi^{2 x}(a, b)-(-b)^{x}}
\end{aligned}
$$

The symmetric property is also satisfied, as we have for Fibonacci and Lucas hyperbolics sine and cosine. For example

$$
\operatorname{TANP}_{x}(a, b)=\frac{\phi^{2 x}(a, b)-(-b)^{x}}{\phi^{2 x}(a, b)+(-b)^{x}}=-\operatorname{TANP}_{-x}(a, b)
$$

Similar to the De'Moivre formulas for the double parameter generalized hyperbolic functions following relation is satisfied

$$
\begin{aligned}
& {\left[C P_{x}(a, b) \pm S P_{x}(a, b)\right]^{n}} \\
& =\left(\frac{2}{\sqrt{a^{2}+4 b}}\right)^{n-1}\left[C P_{n x}(a, b) \pm S P_{n x}(a, b)\right]
\end{aligned}
$$

\section{Generalized Double Parameter Matrices of $n$}

Hoggatt [3] introduced the following Q-matrix and Theory of Fibonacci Q-matrix

$$
Q=\left(\begin{array}{ll}
1 & 1 \\
1 & 0
\end{array}\right)
$$

where $Q^{n}$ connects to the Fibonacci numbers by the fol- lowing relation

$$
Q^{n}=\left(\begin{array}{cc}
F(n+1) & F(n) \\
F(n) & F(n-1)
\end{array}\right)
$$

and

$$
\begin{aligned}
& Q^{-2 n}=\left(\begin{array}{cc}
F(2 n-1) & -F(2 n) \\
-F(2 n) & F(2 n+1)
\end{array}\right) \\
& Q^{-(2 n+1)}=\left(\begin{array}{cc}
-F(2 n) & F(2 n+1) \\
F(2 n+1) & -F(2 n+2)
\end{array}\right)
\end{aligned}
$$

The $G_{a, b}$ matrix of order $(a, b)$ is

$$
G_{a, b}=\left(\begin{array}{ll}
a & 1 \\
b & 0
\end{array}\right)
$$

then for any integer $n=0, \pm 1, \pm 2, \cdots$ the $n$th power of $G_{a, b}$ matrix sets a connection with the two parameter golden number and two parameter Fibonacci numbers such that

$$
\begin{aligned}
G_{a, b}{ }^{n} & =\left(\begin{array}{cc}
P_{n+1}(a, b) & P_{n}(a, b) \\
P_{n}(a, b) & P_{n-1}(a, b)
\end{array}\right) \\
G_{a, b}{ }^{-n} & =\frac{1}{(-b)^{n}}\left(\begin{array}{cc}
P_{n-1}(a, b) & -P_{n}(a, b) \\
-P_{n}(a, b) & P_{n+1}(a, b)
\end{array}\right)
\end{aligned}
$$

where

$$
\operatorname{Det}\left(G_{a, b}^{n}\right)=(-b)^{n}
$$

the procedure can be extended to the case of multi parameters, and we guess

$$
G_{a, 0, \cdots, b, b}=\left(\begin{array}{cccccc}
a & 1 & 0 & 0 & \cdots & 0 \\
0 & 0 & 1 & 0 & \cdots & 0 \\
\vdots & & & \ddots & & \\
0 & 0 & 0 & 0 & \cdots & 1 \\
b & 0 & 0 & 0 & \cdots & 0
\end{array}\right)
$$

and also it maybe generalized to the multi dimension case which allows developing the application to the communication engineering specially to the coding theory $[4,5]$

$$
G_{a_{1}, \cdots, a_{k}}=\left(\begin{array}{cccccc}
a_{1} & 1 & 0 & 0 & \cdots & 0 \\
a_{2} & 0 & 1 & 0 & \cdots & 0 \\
\vdots & & & \ddots & & \\
a_{k-1} & 0 & 0 & 0 & \cdots & 1 \\
a_{k} & 0 & 0 & 0 & \cdots & 0
\end{array}\right)
$$

The inverse can also be calculated similar to the $G_{a, b}$ by induction.

\section{Conclusions}

In this paper the generalized golden ratio is extended to 
the two parameters case and the properties are also generalized to the related historical and recently developed problems. The applications in engineering and science recommended in research works are valid and can be extended to the general cases.

\section{References}

[1] D. H. Fowler, "A Generalization of the Golden Section," Fibonacci Quarterly, Vol. 20, 1982, pp. 146-158.

[2] A. P. Stakhov, "The Generalized Principle of the Golden Section and Its Applications in Mathematics, Science and Engineering," Chaos, Solitons and Fractals, Vol. 26, No. 2, 2005, pp. 263-289. doi:10.1016/j.chaos.2005.01.038

[3] A. Stakhov, "Fundamentals of a New Kind of Mathematics Based on the Golden Section," Chaos, Solitons and Fractals, Vol. 27, No. 5, 2006, pp. 1124-1146. doi:10.1016/j.chaos.2005.05.008

[4] M. Basu and B. Prasad, "Coding Theory on the M-Extension of the Fibonacci P-Numbers," Chaos, Solitons and Fractals, Vol. 42, No. 4, 2009, pp. 2522-2530. doi:10.1016/j.chaos.2009.03.197

[5] M. Basu and B. Prasad, "The Generalized Relations among the Code Elements for Fibonacci Coding Theory," Chaos, Solitons and Fractals, Vol. 41, No. 5, 2009, pp. 2517-2525. doi:10.1016/j.chaos.2008.09.030

[6] E. G. Kocer, N. Tuglu and A. Stakhov, "On the M-Extension of the Fibonacci and Lucas P-Numbers, Chaos," Chaos, Solitons and Fractals, Vol. 40, No. 4, 2009, pp. 1890-1906. doi:10.1016/i.chaos.2007.09.071

[7] J. Veladimirov, "Fundamental Physics," Philosophy and Religion, Kostroma, 1996.

[8] E. Naschie, "On a Class of General Theories for High Energy Particle Physics," Chaos, Solitons and Fractals, Vol. 14, No. 4, 2005, pp. 649-680. doi:10.1016/S0960-0779(02)00033-4

[9] V. V. Petrunenko, "The Golden Section of Quantom States and Its Astronomical and Phisical Manifestations," Pravo i economika, 2005.

[10] Y. S. Vladimirov, "Metaphysics," Binom, Moscow, 2002.

[11] E. Kilic and A. P. Stakhov, "On the Fibonacci and Lucas p-numbers, Their Sums, Families of Bipartite Graphs and Permanents of Certain Matrices," Chaos, Solitons and Fractals, Vol. 40, No. 5, 2009, pp. 2210-2221. doi:10.1016/j.chaos.2007.10.007

[12] A. Stakhov and B. Rozin, "The Continuous Functions for the Fibonacci and Lucas P-Numbers," Chaos, Solitons and Fractals, Vol. 28, No. 4 , 2006, pp. 1014-1025. doi:10.1016/j.chaos.2005.08.158

[13] M. Akbulak and D. Bozkurt, "On the Order- $M$ Generalized Fibonacci K-Numbers," Chaos, Solitons and Fractals, Vol. 42, No. 3, 2009, pp. 1347-1355. doi:10.1016/j.chaos.2009.03.019

[14] A. Stakhov and B. Rozin, "Theory of Binet for Fibonacci and Lucas p-Numbers," Chaos, Solitons and Fractals, Vol. 27, No. 5, 2006, pp. 1162-1177. doi:10.1016/j.chaos.2005.04.106

[15] A. Nalli and P. Haukkanen, "On Generalized Fibonacci and Lucas Polynomials," Chaos, Solitons and Fractals, Vol. 42, No. 5, 2009, pp. 3179-3186. doi:10.1016/j.chaos.2009.04.048

[16] F. Buyukkilic and D. Demirhan, "Cumulative Growth with Fibonacci Approach, Golden Section and Physics," Chaos, Solitons and Fractals, Vol. 42, No. 1, 2009, pp. 24-32. doi:10.1016/j.chaos.2008.10.023

[17] A. Benavoli, L. Chisci and A. Farina, "Fibonacci Sequence, Golden Section, Kalman Filter and Optimal Control," Signal Processing, Vol. 89, No. 8, 2009, pp. 1483-1488. doi:10.1016/j.sigpro.2009.02.003

[18] E. Cureg and A. Mukherjea, "Numerical Results on Some Generalized Random Fibonacci Sequences," Computer and Mathematics Application, Vol. 59, No. 1, 2010, pp. 233-246. doi:10.1016/j.camwa.2009.08.001

[19] E. M. Soroko, "Structural Harmony of System," Nauka i Tekhnika, Russian, 1984.

[20] A. Stakhov and B. Rozin, "The Golden Shofar," Chaos, Solitons and Fractals, Vol. 26, No. 3, 2005, pp. 677-684. doi:10.1016/j.chaos.2005.01.057

[21] A. P. Stakhov, "Generalized Golden Section and a New Approach to Geometric Definition of a Number," Ukrainian Mathematical Journal, Vol. 56, No. 8, 2004, pp. 1143-1150. doi:10.1007/s11253-005-0064-3

[22] O. Karpenkov, "On Examples of Two-Dimensinal Priodic Continued Fractions," Academic Paper, Moscow State University, Moscow, 2004.

[23] M. Grundland, J. Patera, Z. Masakova and N. Dodgson, "Image Sampling with Quasicrystals," Electrical and Electronic Engineering, Vol. 5, 2009, pp. 1-23. doi: 10.3842/SIGMA.2009.075

[24] S. Dutch, "Why Trisecting of the Angle Is Impossible?" Natural and Applied Sciense, University of Wisconsin-Green Bay, Green Bay, 2009.

[25] J. B. Fraleigh, "A First Course in Abstract Algebra," 7th Edition, Addison-Wesley, Boston, 1982.

[26] M. B. Farnworth, "Cubic Equations and Ideal Trisection of the Arbitrary Angle," Theaching Mathematics and Its Applications, Vol. 25, No. 2, 2006.

[27] S. Falcon and A. Plaza, "On the Fibonacci K-Numbers," Chaos, Solitons and Fractals, Vol. 32, No. 5, 2007, pp. 1615-1624. doi:10.1016/j.chaos.2006.09.022

[28] A. Stakhov, "The Golden Section, Secret of the Egyption Civilization and Harmony Mathematics," Chaos, Solitons and Fractals, Vol. 30, No. 2, 2006, pp. 490-505. doi:10.1016/j.chaos.2005.11.022 